\title{
IL-6 secreted by cancer-associated fibroblasts promotes epithelial-mesenchymal transition and metastasis of gastric cancer via JAK2/STAT3 signaling pathway
}

\author{
Xiongyan Wu $\mathbf{u}^{1, *}$, Pan Tao ${ }^{1, *}$, Quan Zhou ${ }^{1, *}$, Jie $\mathrm{Li}^{1}$, Zhenjia $\mathbf{Y u}^{1}{ }^{1}$, Xiaofeng Wang ${ }^{1}$, \\ Jiaanfang Li ${ }^{1}$, Chen Li ${ }^{1}$, Min Yan ${ }^{1}$, Zhenggang Zhu ${ }^{1}$, Bingya Liu ${ }^{1}$, Liping Su${ }^{1}$ \\ ${ }^{1}$ Department of Surgery, Shanghai Key Laboratory of Gastric Neoplasms, Shanghai Institute of Digestive Surgery, Ruijin \\ Hospital, School of Medicine, Shanghai Jiao Tong University, Shanghai 200025, People's Republic of China \\ *These authors contributed equally to this work
}

Correspondence to: Liping Su, email: suliping@shsmu.edu.cn

Keywords: cancer-associated fibroblasts, interleukin-6, JAK/STAT3, gastric cancer

Received: July 22, $2016 \quad$ Accepted: January 23, 2017

Published: February 06, 2017

\section{ABSTRACT}

Cancer-associated fibroblasts (CAFs), as the activated fibroblasts in tumor stroma, are important modifiers of tumor progression. However, the molecular mechanisms underlying the tumor-promoting properties of CAFs in gastric cancer remain unclear. Here, we show that CAFs isolated from gastric cancer produce significant amounts of interleukin-6 (IL-6). CAFs enhances the migration and EMT of gastric cancer cells through the secretion of IL-6 that activates Janus kinase 2/signal transducers and activators of transcription (JAK2/STAT3) pathway in gastric cancer cells, while deprivation of IL-6 using a neutralizing antibody or inhibition of JAK/STAT3 pathway with specific inhibitor AG490 markedly attenuates these phenotypes in gastric cancer cells induced by CAFs. Moreover, silencing IL-6 expression in CAFs or inhibiting JAK2/STAT3 pathway in gastric cancer cells impairs tumor peritoneal metastasis induced by CAFs in vivo. Taken together, these results suggest that CAFs in the tumor microenvironment promote the progression of gastric cancer through IL-6/JAK2/STAT3 signaling, and IL-6 targeted therapy could be a complementary approach against gastric cancer by exerting their action on stromal fibroblasts.

\section{INTRODUCTION}

Gastric cancer, one of the most common primary malignant tumors, is the third leading cause of cancer death in worldwide with the highest estimated mortality rates in Eastern Asia [1]. Despite advances in early detection, diagnosis, and treatment of gastric cancer, the overall prognosis is still poor and the 5-year survival for patients with gastric cancer has remained 20-25\%, which is due to the recurrence and metastasis after surgery [2]. Thus, a better understanding of the molecular mechanisms in promoting the progression of gastric cancer will be helpful to improve the prevention, diagnosis, and treatment of gastric cancer.

Epithelial-mesenchymal transition (EMT), a wellcharacterized embryological process, has been identified to play a critical role in tumor progression, including invasion and metastasis, by which cancer cells could gain more aggressive properties. In the process of EMT, epithelial cells undergo a phenotypic switch by lossing their cell polarity and the epithelial markers (E-cadherin, $\beta$-catenin), to form mesenchymal cells through acquiring the mesenchymal markers (N-cadherin, vimentin, ZEB2), thus these transformed epithelial cells acquire fibroblastlike properties and exhibit reduced cell-cell adhesion and increased motility [3-5]. The enhanced motility and invasiveness afforded by EMT is critical in the initiation of metastasis for cancer progression, and the acquisition of a mesenchymal phenotype has been also enhanced resistance to chemotherapy and poor prognosis $[6,7]$.The expression of these EMT markers can be induced by a number of growth factors/cytokines such as hepatocyte growth factor (HGF), transforming growth factor (TGF)- $\beta$, CXCL12 and hypoxia-inducible factor-1 $\alpha$ (HIF-1 $\alpha$ ) [8-11].

Accumulating evidence has indicated that interactions between tumor and stromal cells create a unique microenvironment that is essential for tumor growth, invasion, and metastasis [12, 13]. Therefore, epithelial cellstromal cell interactions usually act as the regulators of EMT, and the factors inciting EMTs are often originated from the 
stromal cells creating the tumor microenvironment. Cancerassociated fibroblasts (CAFs), the important components of tumor stroma, are the key players in regulating tumor progression $[12,13]$. It has been observed that CAFs actively communicate with cancer cells through growth factors or inflammatory cytokines such as HGF, IL-6, TGF- $\beta$, VEGF, FGF, and CXCL12 that can promote tumorigenesis and progression [12-16]. IL-6 is a multifunctional cytokine that was originally determined to be a regulator of immune and inflammatory responses [17] and has been also reported to be associated with certain epithelial tumors such as colon cancer, prostate cancer $[18,19]$. Aberrant production and signaling of IL-6 is tightly linked to tumor generation and poor disease outcome in many cancer types, including gastric cancer. IL- 6 exerts its effects by binding to IL- $6 \alpha$ chain and a common cytokine receptor signal-transducing subunit gp130, which leads to activation of the Janus kinases (JAKs) family of tyrosine kinases and the signal transducers and activators of transcription (STAT) family, particularly STAT3 [20]. Activation of IL-6-JAK-STAT3 signaling pathway plays an active role in the oncogenesis of a variety of tumors [21-23]. Previous studies have demonstrated that STAT3 is activated in gastric cancer tissues. Menheniott TR, et al. detected STAT3 in antral biopsies, and have demonstrated that both total STAT3 and phosphorylated STAT3 increased in intestinal-type gastric cancer compared with normal stomach. Zhang XM et al also found that activated STAT3 is positive in early gastric cancer, poorly differentiated adenocarcinoma and metastatic lymph node tissues [24, 25]. However, the role of CAFs and IL-6 in gastric cancer has not been well addressed. Therefore, our aim was to determine how CAFs can enhance tumor metastasis and EMT changes of gastric cancer cells, and link CAFs with activation of the IL-6-JAKSTAT3 signaling pathway in the progression of gastric cancer. In this study, we find that CAFs isolated from gastric cancer produce considerable amounts of IL-6. CAFs-derived IL-6 enhances the migration and EMT of gastric cancer cells by the activation of JAK2/STAT3 pathway in gastric cancer cells, while deprivation of IL-6 using a neutralizing antibody or inhibition of JAK2/STAT3 pathway with specific inhibitor AG490 markedly reduces these phenotypes in gastric cancer cells induced by CAFs. Moreover, knockdown the expression of IL-6 in CAFs by RNAi or inhibiting JAK2/ STAT3 pathway in gastric cancer cells by the specific inhibitor AG490 significantly retards the tumor peritoneal metastasis induced by CAFs in vivo. These results suggest that suppressing IL-6 or its downstream targets could serve as an effective therapeutic strategy against gastric cancer by exerting their action on stromal fibroblasts.

\section{RESULTS}

\section{IL-6 is highly expressed in CAFs in the tumor microenvironment of gastric cancer}

Cancer-associated stroma secretes a plethora of factors such as HGF, IL-6, TGF- $\beta$ to promote the growth and invasion of the underlying tumor [16]. It has been reported that IL-6 is associated with certain epithelial tumors. To determine the expression level and cellular source of IL-6 in gastric cancer, we first detected IL-6 expression in both serum and cancer tissues in gastric cancer patient by ELISA. As shown in Figure 1A, IL-6 levels in the serum of gastric cancer patients were significantly elevated in comparison with healthy volunteers. Similarly, the expression level of IL-6 was also significantly higher in the cancer tissues compared with adjacent non-cancerous tissues (Figure 1B). To further illustrate which cell component(s) in gastric cancer tissues is (are) responsible for the high expression of IL-6, we detected IL-6 expression in tissues by immunofluorescence staining. As shown in Figure 1C, the expression of IL-6 was noted in stromal cells of periand intra-tumoral areas rather than in the cytoplasm or the nucleus of the cancer cells. Anti $\alpha$-SMA antibody are used to identify CAFs. Upon immunofluorescence staining, we identified both $\alpha$-SMA and IL- 6 expressing stromal cells in peri- and intra-tumoral areas (Figure 1C). We further quantified expression levels of IL-6 in CAFs, normal fibroblasts (NFs) and gastric cancer cells (SNU-1, MKN45, SGC7901 and MKN28) by ELISA. We found IL-6 level were significantly higher in CAFs compared to NFs and gastric cancer cells (Figure 1D). These findings suggest that IL-6 is overexpressed in gastric cancer and CAFs is one major cell source in producing IL-6 in the tumor microenvironment of gastric cancer.

\section{CAFs enhance the migration of gastric cancer cells via the secretion of IL-6}

We examined the migration of gastric cancer cells induced by CAFs, a key determinant of metastasis in tumor progression. As shown in Figure 2A and 2B, SGC7901 cells co-cultured with CAFs showed enhanced ability of migration than SGC-7901 cells alone (SGC7901 cells alone, $15.8 \pm 5.0$ cells per field; SGC-7901 cells co-cultured with CAFs, $156.4 \pm 55.5$ cells per field; $P<0.01)$. However, adding neutralizing IL-6 antibody into the co-culture system led to significantly decreased migration (neutralizing IL-6 group, $93.6 \pm 24.9$ cells per field; isotype control group, $179.0 \pm 41.9$ cells per field; $P<0.01)$ of SGC-7901 cells. Similarly, MKN28 cells co-cultured with CAFs also exhibited higher ability of migration than MKN28 cells alone (MKN28 cells alone, $10.6 \pm 7.3$ cells per field; MKN28 cells co-cultured with CAFs, $48.8 \pm 15.4$ cells per field; $P<0.01$ ), while the migratory ability of MKN28 cells co-cultured with CAFs was significantly reduced by the addition of anti-IL-6 neutralizing antibody (neutralizing IL-6 group, $27.2 \pm$ 17.7 cells per field; isotype control group, $53.0 \pm 20.8$ cells per field; $P<0.01$ ) (Figure $2 \mathrm{~A}$ and $2 \mathrm{C}$ ). Moreover, we determined the migration ability of gastric cancer cells induced by exogenous IL-6. Gastric cancer cells stimulated by IL- 6 showed enhanced ability of migration 
compared with gastric cancer cells alone (Supplementary Figure 1). Thus, these data suggest that CAFs enhance the migration of gastric cancer cells via the secretion of IL-6.

\section{CAFs promote EMT changes of gastric cancer cells via the secretion of IL-6}

EMT, a well-characterized embryological process, has been identified to play a critical role in tumor metastasis, which is characterized by losing epithelial markers (e.g. E-cadherin), and acquiring of mesenchymal markers (e.g. N-cadherin, ZEB2) [5].To examine the role of CAFs in mediating EMT in gastric cancer cells, we cultured SGC-7901 cells or MKN28 cells with CAFs in a previous described co-culture system [14]. As shown in
Figure 3A and 3B, co-culture of CAFs with SGC-7901 cells markedly decreased the expression of E-cadherin and simultaneously increased the expression of $\mathrm{N}$-cadherin and ZEB2 in SGC-7901 cells. The similar phenotype changes of EMT were also occurred in MKN28 cells after co-cultured with CAFs (Figure $3 \mathrm{~A}$ and $3 \mathrm{~B}$ ). To determine whether IL-6 contributes to the EMT effect of CAFs on gastric cancer cells, we added the IL-6 neutralizing antibody into the co-culture system. As shown in Figure $3 \mathrm{~A}$ and $3 \mathrm{~B}$, EMT induced by CAFs was impaired when the IL-6 was abolished by its specific neutralizing antibody, which was demonstrated by inhibition of E-cadherin decrease, and N-cadherin or ZEB2 increase. These data suggest that CAFs promote the EMT of gastric cancer cells via the secretion of IL-6.
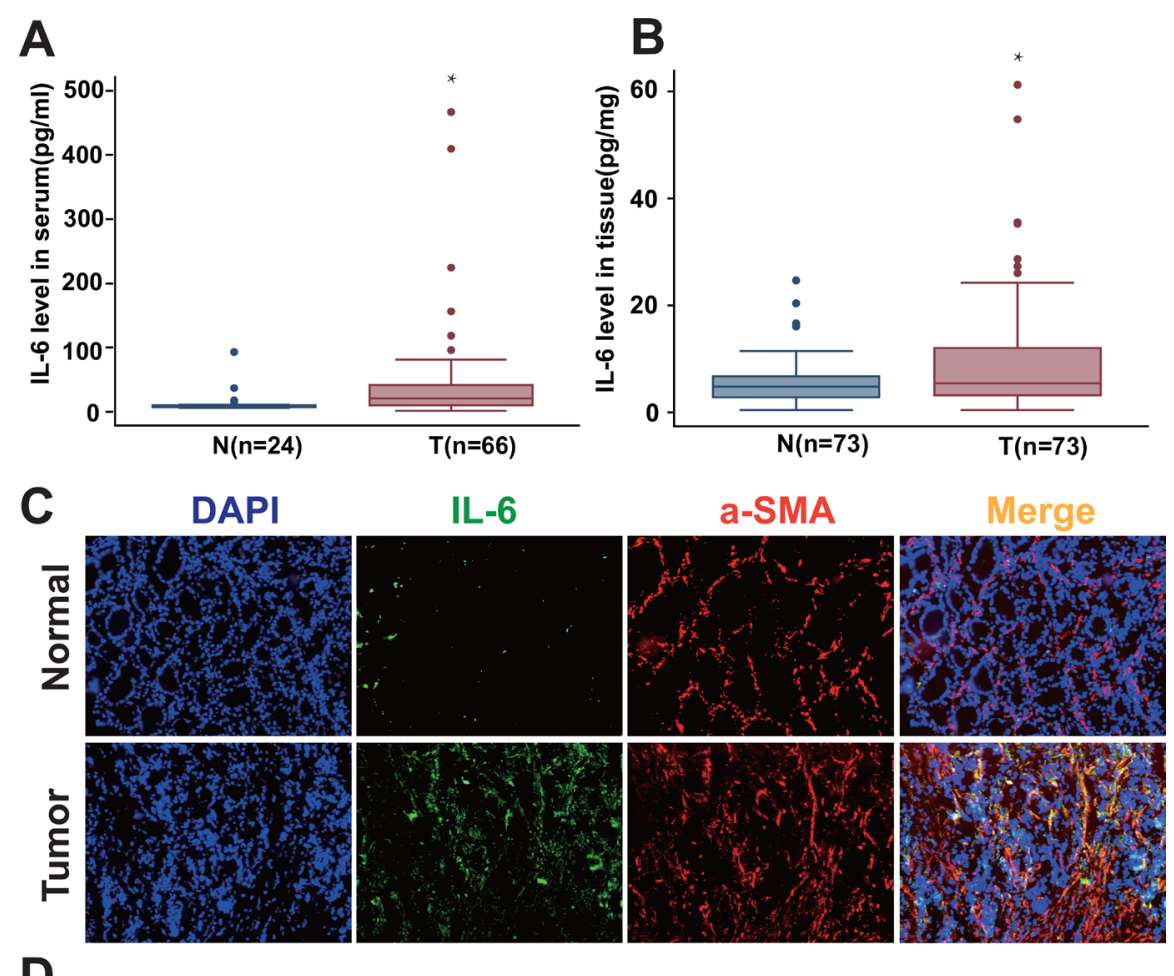

D

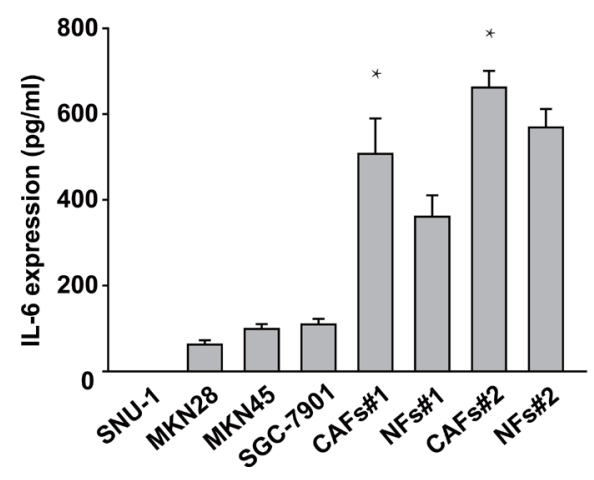

Figure 1: IL-6 is highly expressed in gastric CAFs. (A, B) The expression of IL-6 in both serum and cancer tissues in gastric cancer patient was detected by ELISA. (C) Frozen tissue sections from gastric cancer and adjacent non-tumor tissue were immunostained by DAPI (nucleus), FITC (IL-6) and PE ( $\alpha$-SMA) $(200 \times)$. (D) IL-6 protein expression level in the gastric cancer cells (SNU-1, MKN45, SGC7901 and MKN28), gastric CAFs and normal fibroblasts (NFs) was quantified $24 \mathrm{hrs}$ after change the culture medium by ELISA. $* P<0.05$. 

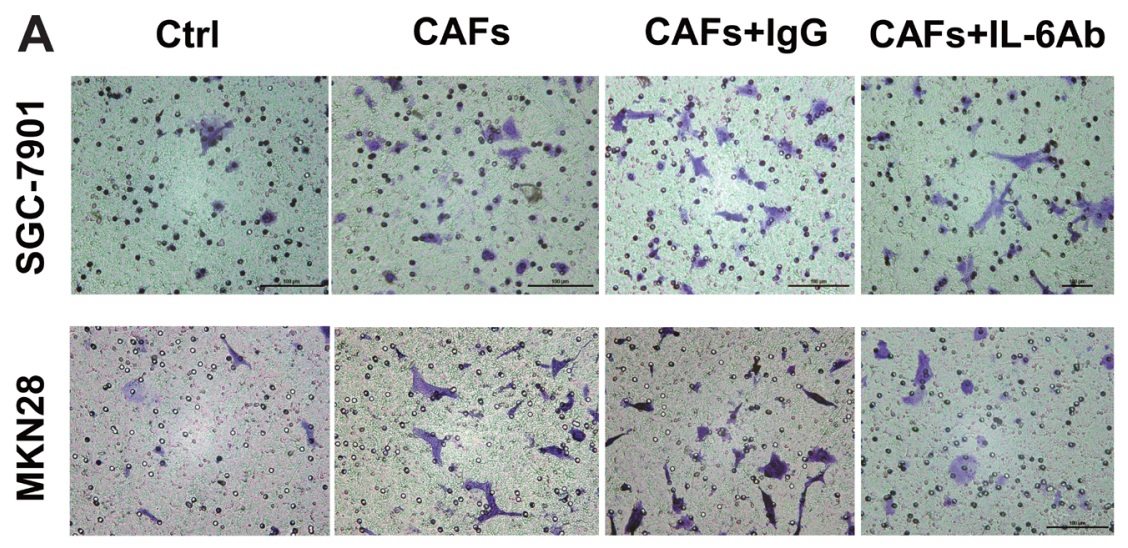

B

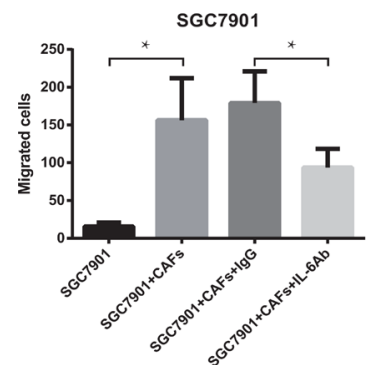

C

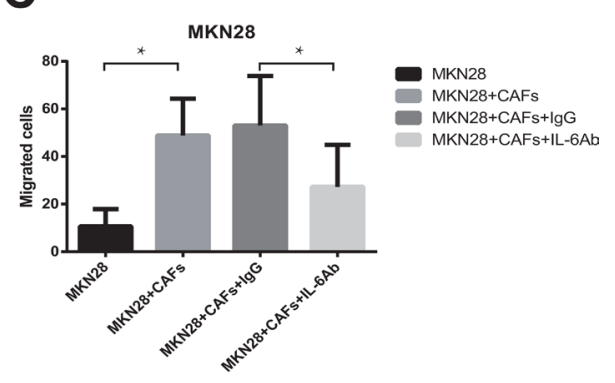

Figure 2: CAFs enhance the migration of gastric cancer cells via the secretion of IL-6. (A) The effect of CAFs on cell migration was determined $24 \mathrm{hrs}$ after in the presence of IL-6 neutralizing antibody or IgG isotype control antibody. Representative photographs of migratory cells on the membrane (magnification, 100×) are shown. (B, C) Migratory Cells were counted in ten randomly selected microscopic fields. Values are represented as mean $\pm \mathrm{SD}$ of three independent experiments. $* P<0.05$.

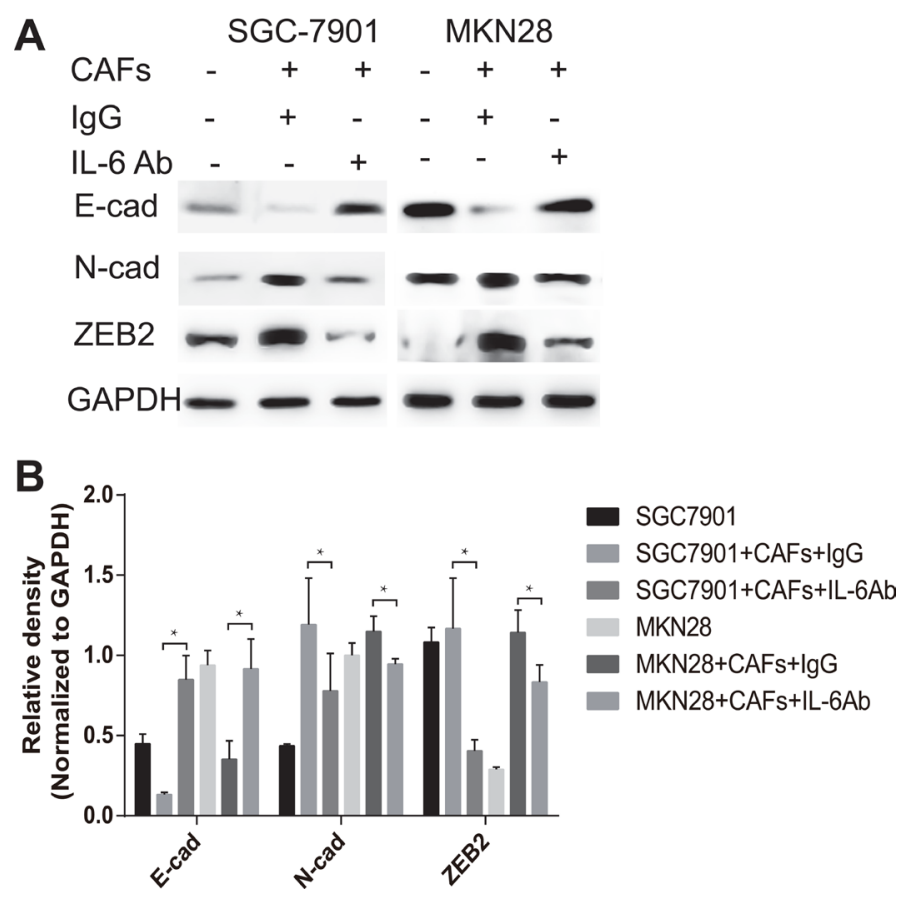

Figure 3: CAFs promote EMT of gastric cancer cells via the secretion of IL-6. (A) Protein expression of E-cadherin, N-cadherin and ZEB2 in gastric cancer cells SGC-7901 and MKN28 co-cultured with CAFs in the presence of IL-6 neutralizing antibody or IgG isotype control antibody was analyzed by western blot. Representative images from one of the three independent experiments are presented. (B) Densitometric analysis of E-cadherin, N-cadherin and ZEB2 expression is shown. 


\section{CAFs-derived IL-6 mediates the migration and EMT of gastric cancer cells via the activation of JAK2/STAT3 pathway}

The canonical IL-6 signal transduction pathway is initiated by binding to IL-6R and phosphorylation STAT3 through the activation of JAK2. To interrogate the role of IL-6-JAK2-STAT3 pathway in mediating CAFs-induced migration and EMT changes of gastric cancer cells, we first explored the activation of IL-6-JAK2-STAT3 pathway in gastric cancer cells after co-culture with CAFs. As shown in Figure 4A-4D, CAFs significantly induced the phosphorylation of JAK2 and STAT3 in both SGC7901 cells and MKN28 cells. In contrast, adding IL-6 neutralizing antibody or JAK-2 protein tyrosine kinase inhibitor AG490 into the co-culture system significantly reversed CAF-mediated phosphorylation of JAK2 and STAT3 in gastric cancer cells (Figure 4A-4D). We further examined whether block the JAK2/STAT3 pathway by AG490 could also inhibit the tumor-promoting effects on gastric cancer cells via the secretion of IL-6 by CAFs. As shown in Figure 4B and 4D, inhibition of JAK2-STAT3
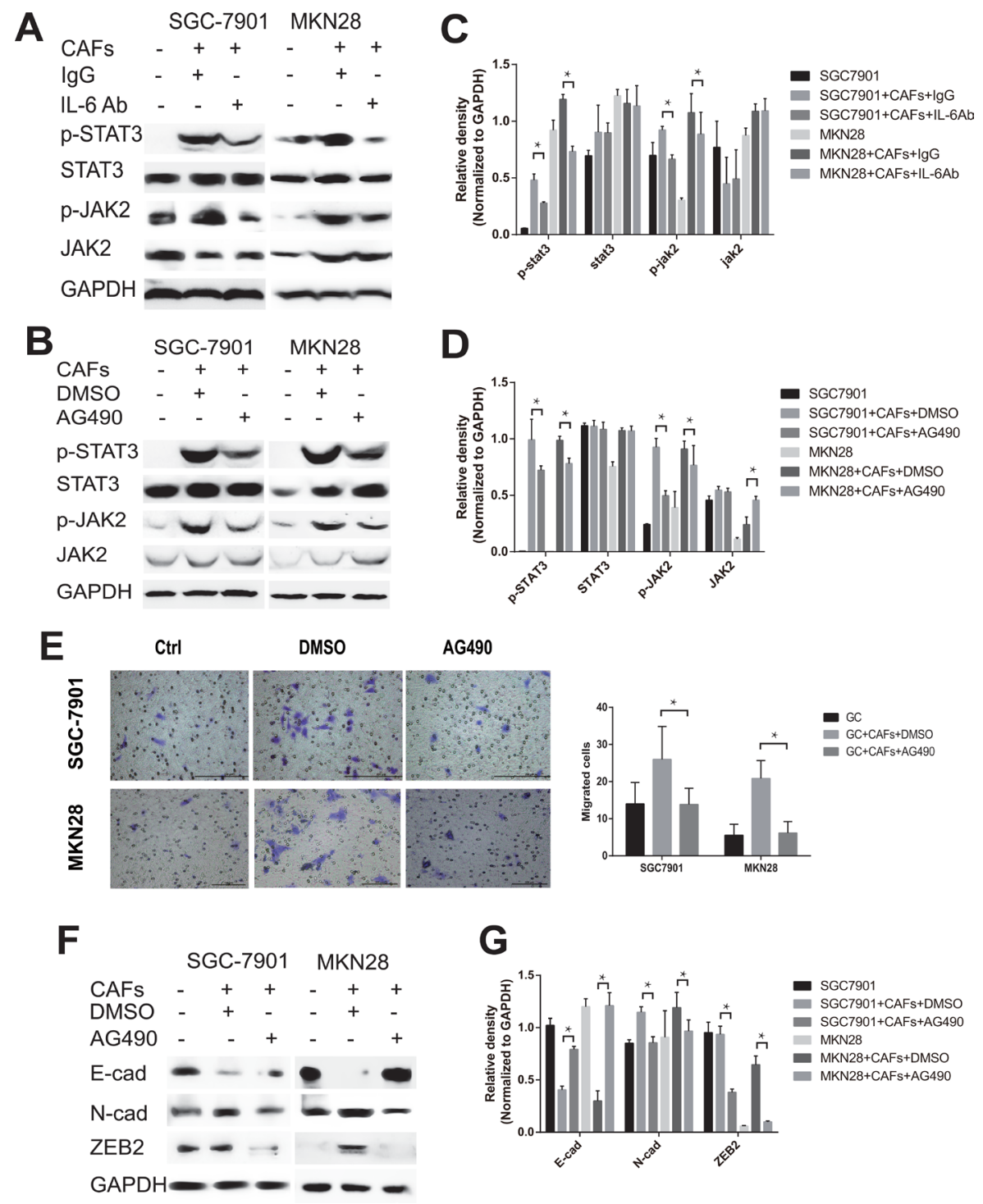

Figure 4: CAFs-derived IL-6 enhances the migration and EMT of gastric cancer cells via the activation of JAK2/ STAT3 pathway. (A, B) Protein levels of p-STAT3, STAT3, p-JAK2 and JAK2 in both SGC-7901 and MKN28 co-cultured with CAFs in the presence of IL-6 neutralizing antibody or AG490 were analyzed by western blot. Representative results from one of the three independent experiments are presented. (C, D) Densitometric analysis of p-STAT3, STAT3, p-JAK2 and JAK2 expression is shown. (E) The effect of CAFs on cell migration was assayed in the presence of AG490. Migratory Cells were counted in ten randomly selected microscopic fields. Values are represented as mean \pm SD of three independent experiments. ${ }^{*} P<0.05$. (F) Protein expression of E-cadherin, $\mathrm{N}$-cadherin and ZEB2 in gastric cancer cells SGC-7901 and MKN28 co-cultured with CAFs in the presence of AG490 or equivalent concentration of DMSO was analyzed by western blot. Representative images from one of the three independent experiments are presented. (G) Densitometric analysis of E-cadherin, N-cadherin and ZEB2 expression is shown. 
pathway activation by AG490 significantly dampened cell migration (Figure 4E) and EMT (Figure 4F and 4G) induced by CAFs. These results indicate that IL-6-JAK2STAT3 signal pathway plays an important role in CAFsinduced migration and EMT of gastric cancer cells.

\section{Blocking IL-6-JAK2-STAT3 pathway impairs the peritoneal dissemination and metastasis of gastric cancer cells induced by CAFs in vivo}

Peritoneal metastasis is a prevalent form of recurrence and metastasis in advanced gastric cancer. We further determined the contribution of IL-6-JAK2-STAT3 pathway to the peritoneal dissemination and metastasis of gastric cancer cells in vivo. CAFs were transiently transfected with human IL-6 siRNA (silL-6) or with control scrambled siRNA (siNC). Knockdown of IL-6 expression in CAFs with IL-6 siRNA was confirmed by qRT-PCR and ELISA assay (Figure 5A and 5B). We then mixed CAFs with gastric cancer cells in a 1:4 ratio and intraperitoneally inoculated them into immunodeficient nude mice. As shown in Figure 5C and 5D, SGC-7901 cells mixed with CAFs-silL-6 developed fewer peritoneal metastases than those developed by SGC-7901 cells mixed with CAFs-siNC $(P<0.05)$. We next examined whether inhibiting the activation of JAK2-STAT3 pathway by AG490 could also impair peritoneal metastasis induced by CAFs in vivo. We observed fewer peritoneal metastases of the CAFs/SGC-7901/AG490 group but not in CAFs/SGC7901 group (Figure 5C and 5D). These results indicate
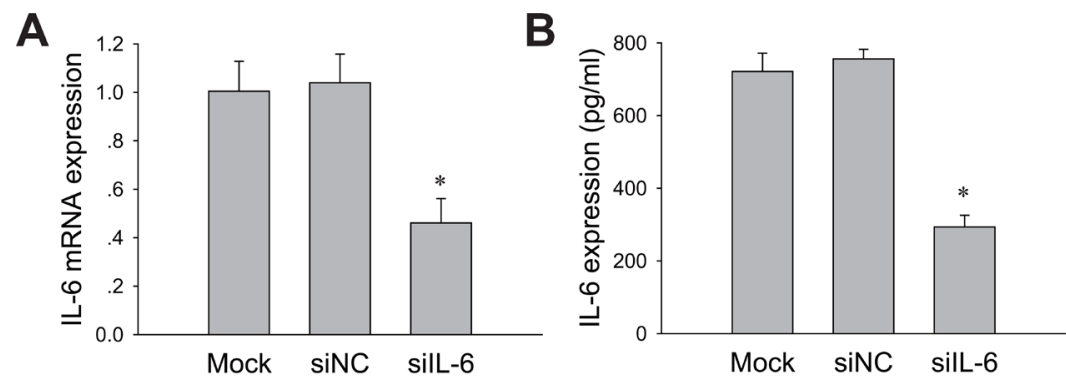

C

SGC-7901
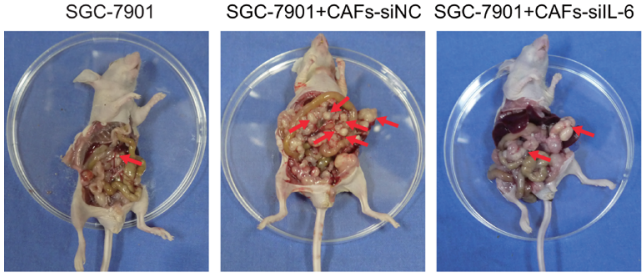

D

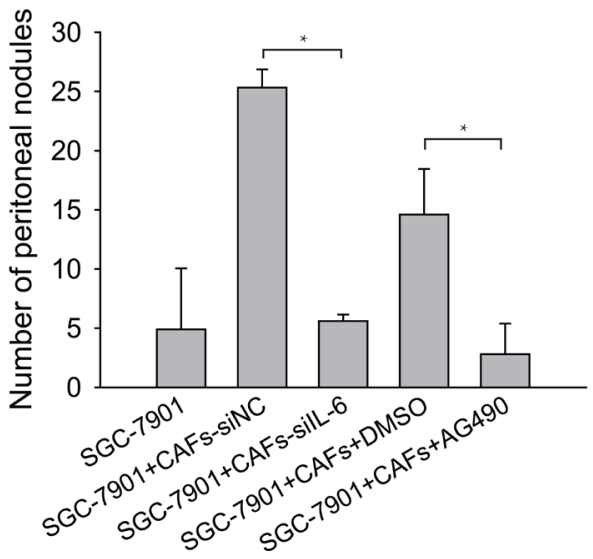

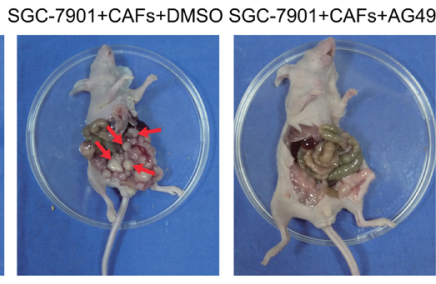

5: Blocking IL-6-JAK2-STAT3 pathway impairs tumor peritoneal dissemination and metastasis induced by CAFs in vivo. (A) IL-6 mRNA levels in CAFs treated with siRNA or siNC were detected by qRT-PCR. (B) IL-6 protein concentration in the medium conditioned by CAFs treated with (silL-6) or control siRNA (siNC) were measured by ELISA. (C) SGC-7901 cells and SGC-7901 cells mixed with CAFs that were transfected with IL-6 siRNA (siIL-6) or control siRNA (siNC) were inoculated into nude mice. Mice co-injected with SGC-7901 cells and CAFs were administered i. $p$ with AG490 (500 $\mu \mathrm{g} / 100 \mathrm{ul} / \mathrm{mouse})$ or equivalent concentration of DMSO once a week. The peritoneal nodules (red arrows) were observed after 30 days ( $N=5$ per group). (D) Average peritoneal nodules from nude mice are shown. Data are representative of three independent experiments. $* P<0.05$. 
that IL-6-JAK2-STAT3 pathway is required for peritoneal dissemination and metastasis of gastric cancer cells induced by CAFs in vivo, and pharmacologic blocking IL-6/JAK2/STAT3 signaling pathway in gastric cancer could reduce peritoneal dissemination and metastasis induced by CAFs.

\section{DISCUSSION}

CAFs, the activated fibroblasts in cancer stroma, are the most abundant cells in the tumor microenvironment. Accumulating evidences demonstrate that CAFs play a prominent role in tumor growth and progression, and could be a promising tool to cancer therapeutics [26]. Therefore, a better understanding of the molecular mechanism for the tumor-promoting prosperities of CAFs is of obvious importance for understanding in gastric cancer progression and finding novel strategies to it. In this study, we demonstrate that IL-6 secreted by CAFs plays an important role in the progression of gastric cancer. We show that CAFs derived-IL-6 promoted the migration and EMT of gastric cancer cells via the activation of JAK2STAT3 pathway, and blocking this pathway with IL-6 neutralizing antibody or JAK2 specific inhibitor AG490 impaired gastric cancer metastasis induced by CAFs in vivo. Therefore, the activation of JAK2-STAT3 pathway by IL-6 may play a central role in the interplay between CAFs and gastric cancer cells.

Previous studies have shown that cancer-associated stroma secretes a plethora of factors which promote the growth and invasion of the underlying tumor [16]. IL-6 is a multifunctional cytokine that was originally determined to be a regulator of immune and inflammatory responses [17]. Increasing evidences have suggested that IL-6 plays a critical role in modulating the function and activity of tumor-associated immune cells. For example, IL-6 inhibits the differentiation of dendritic cells, activates a Th17 cell response promoting tumorigenesis and expands myeloid derived suppressor cells (MDSC) [27]. In contrast to the effects of IL-6 on tumor-associated immune cells, IL-6 is also a direct critical driver of tumor growth and metastasis. Recent studies have shown that aberrant expression of IL-6 is tightly linked to tumor generation and poor disease outcome in many cancer types, including gastric cancer $[18,19,28]$. In this study, we reveal that IL-6 was overexpressed in both serum and cancer tissue of gastric cancer patients, and significantly higher expression was found in stromal fibroblasts. These results indicate CAFs is the major cell source in producing IL-6 in the tumor microenvironment of gastric cancer.

Interestingly, although NFs secreted lower IL-6 than CAFs, it also produced abundant IL-6 and significant higher than that from gastric cancer cells. Cancerassociated fibroblasts can be activated in incipient neoplasia to orchestrate tumor-promoting inflammation in an NF-kB-dependent manner [12, 29, 30]. Tissue-resident fibroblasts are suggested as precursors for CAFs, activated by tumor and immune cell-derived factors to express proinflammatory genes such as IL-1, IL-6, IL-8 and SDF-1. We previously showed that $\mathrm{H}$. pylori infection induces the secretion of PGE2 by gastric epithelial cells that consequently silences stromal miR-149. The silence of miR-149 removes the suppression of its target gene IL-6, leading to elevated level of IL- 6 that acts on fibroblasts to stimulate the transformation of NF into CAF [31]. Thus, cancer cells take advantage of the enormous plasticity of stromal fibroblasts and produce multiple signals that generate a tumor-promoting microenvironment.

EMT is an important embryonic process that is hijacked by tumor cells to facilitate execution of most of the invasion-metastasis cascade. Recently, Yadav et al. have shown that IL-6 induces EMT changes in head and neck tumor by up-regulating snail expression [32]. Breast cancer cells that constitutively expressed Twist, a EMT regulator and direct transcriptional repressor of E-cadherin, exhibited aberrant IL-6 production and STAT3 activation [33]. Initiation of metastasis requires tumor cell migration and invasion, which is enabled by EMT. In the present study, we have shown that IL-6 secreted by CAFs induced EMT of gastric cancer cells, which is characterized by losing epithelial markers E-cadherin and acquiring of mesenchymal markers N-cadherin and ZEB2. Then these EMT changes contribute to the enhanced capability of active locomotion of gastric cancer cells, which is demonstrated by increased migratory ability triggered by CAFs.

IL-6 exerts its effects by binding to a cell-surface type I cytokine receptor complex consisting IL-6a chain (CD126) and a common cytokine receptor signaltransducing subunit gp130, which forms a complex to activate STAT3 with the phosphorylation of Tyr705 via the JAK signaling pathway [20, 34]. Accumulating evidences show that activation of IL-6-JAK2-STAT3 signaling pathway by growth factors or cytokines plays an active role in tumor growth and progression. However, the role of CAFs and IL-6 in gastric cancer has not been well addressed. Our present study has shown that CAFs induced the phosphorylation of JAK2 and STAT3 of gastric cancer cells via the secretion of IL-6, and inhibiting JAK2-STAT3 pathway activation with AG490 significantly impaired cell migration and EMT, as well as peritoneal dissemination and metastasis in vivo induced by CAFs. CAFs are known to secrete multiple growth factors and chemokines such as SDF-1, VEGF, FGF, and CXCL14 into the tumor microenvironment that promote the growth and invasion of the underlying tumor by triggering multiple pathways [14, 35-37]. In the present study, we found that IL-6 neutralizating antibody partly suppressed the STAT3 or JAK2 phosphorylation, which suggested that IL-6 contributed partially to the tumor-promoting effect of CAFs on GC cells. Although we cannot preclude the likely involvement of other growth factors and/or cytokines, the 
studies of neutralizing IL-6 or inhibiting JAK2-STAT3 pathway activation with AG490 reveal that IL-6 is an important mediator in tumor-promoting effects of gastric CAFs that promotes EMT and peritoneal metastasis via the activating JAK2/STAT3 signaling pathway in gastric cancer.

In summary, we find that the crosstalk between gastric cancer cells and their stromal cells-CAFs contributes to tumor progression through IL-6-JAK2STAT3 signaling. Recently, anti-IL6 directed therapies have been used clinically to treat various diseases, such as rheumatoid arthritis [38]. Thus, our results suggest that IL-6 targeted therapy could be a complementary approach against gastric cancer by exerting their action on stromal fibroblasts.

\section{MATERIALS AND METHODS}

\section{Patient samples and cell lines}

Plasma samples were collected from 66 preoperative gastric cancer patients, and 24 age-matched healthy volunteers. Gastric cancer tissues were obtained from 73 patients, between 2009 and 2012 at Ruijin hospital, School of Medicine, Shanghai Jiaotong University. None of the gastric cancer patients received radiotherapy or chemotherapy before surgery. Informed consent was obtained from each patient and healthy control for the use of their blood samples in this study. All the tissue samples were identified by clinical pathologist.

Gastric cancer cell lines SNU-1, MKN45, SGC7901 and MKN28 were purchased from Shanghai Institutes for Biological Sciences, Chinese Academy of Sciences, and were cultured at $37^{\circ} \mathrm{C}$ in a humidified atmosphere of $5 \% \mathrm{CO}_{2}$ with RPMI-1640 medium containing $10 \%$ fetal calf serum with $100 \mathrm{U} / \mathrm{ml}$ penicillin and $100 \mathrm{U} / \mathrm{ml}$ streptomycin. Fibroblasts were isolated from 4 independent gastric cancer patients during radical gastric resection as previous described [14].

\section{Enzyme-linked immunosorbent assay (ELISA)}

The protein levels of IL-6 in plasma and tissue lysates were measured by an ELISA kit (R\&D Systems, Minneapolis, MN, USA) according to the manufacturer's instructions.

\section{Immunofluorescence}

Tissues were fixed in 10\% neutralized formalin and embedded in paraffin blocks. Sections $(4 \mu \mathrm{m})$ were dewaxed with xylene and rehydrated with gradient ethanol, and then gently rinsed with phosphate buffered saline (PBS) for three times. After blocking with normal nonimmune goat serum for $30 \mathrm{~min}$, tissue sections were incubated with anti- $\alpha$-smooth-muscle actin and anti-IL-6 antibodies (Abcam, Cambridge, USA) at $37^{\circ} \mathrm{C}$ for $2 \mathrm{~h}$. After 5 times rinses with PBS, cells were stained with appropriate Alexa dye-conjugated secondary immune reagents, Alexa dye-conjugated phalloidin, and Hoechst 33342 (Invitrogen, Carlsbad, CA, USA). Negative control staining was performed by omission of the primary antibody.

\section{Quantitative real-time PCR (QRT-PCR)}

Total RNA was extracted using Trizol reagent (Invitrogen, Carlsbad, CA, USA) following the manufacturer's manual. RNA $(1 \mu \mathrm{g})$ was reverse transcribed into cDNA using Reverse Transcription system (Promega, Madison, WI, USA). QRT-PCR was performed to quantify IL-6 mRNA level with the SYBR Green PCR core Reagent kit (Applied Biosystems, Foster city, CA, USA). GAPDH was used as the endogenous reference. Data were analyzed by using the comparative $\mathrm{Ct}$ method. Specificity of resulting PCR products was confirmed by melting curves. The primers used in this assay were: IL-6: 5'-CGGTCCAGTTGCCTTCTCCC-3' (upper) and 5'-GAGTGGCTGTCTGTGTGGGG-3' (lower); GAPDH: 5'-GGACCTGACCTGCCGTCTAG-3' (upper) and 5'-GTAGCCCAGGATGCCCTTGA-3' (lower).

\section{Cell migration assay}

Cell migration assays were performed by using $8 \mu \mathrm{m}$ transwell chambers (Corning Life Science, MA, USA). Cells were incubated in serum-free medium for $24 \mathrm{~h}$ and then were added to the upper chamber, and $5 \times 10^{4} \mathrm{CAFs}$ in $500 \mu \mathrm{l}$ in RPMI 1640 medium containing 10\% FBS were added to the lower chamber. Non-migrating cells from the interior of the inserts were removed with cottontipped swabs $48 \mathrm{~h}$ later, and cells that migrated to the bottom of the membranes were stained with $0.1 \%$ crystal violet for $30 \mathrm{~min}$. The stained cells were counted and photographed. At least ten randomly selected fields were counted and the average number was presented.

\section{Western blot analysis}

Cells were lysed with RIPA cell lysis buffer in the presence of protease inhibitor cocktail (Sigma, USA). The same amount of protein samples were loaded onto $10 \%$ SDS-PAGE and then transferred onto PVDF membranes. After blocked by skim milk, the membranes were incubated in the primary antibodies diluted by TBST buffer for overnight at $4{ }^{\circ} \mathrm{C}$ and then in the HRP-conjugated secondary antibody for $2 \mathrm{~h}$ at room temperature. Finally the protein bands images were captured by a Tanon detection system with ECL reagent (Thermo). The primary antibodies used in the experiments were Anti-GADPH, anti-E-cadherin, anti-N-cadherin, and anti-ZEB2 antibodies were purchased from Proteintech. 


\section{RNA interference}

Human IL-6 siRNA and scrambled siRNA (Santa Cruz, CA, USA) at the final concentration of $100 \mathrm{nmol} / \mathrm{L}$ were transfected into CAFs cells with Lipofectamine 2000 reagent (Invitrogen, Carlsbad, CA, USA). Cells were collected for further assay at $24 \mathrm{~h}$ and $48 \mathrm{~h}$ after transfection.

\section{Tumor xenograft model and tumorigenicity assay}

Male BALB/c nu/nu nude mice at the age of 4-5 weeks (Institute of Zoology Chinese Academy of Sciences), were housed at a specific pathogen-free environment in the Animal Laboratory Unit, Shanghai Jiao Tong University School of Medicine, China. Fibroblasts and SGC-7901cells were mixed at the ratio of 1:4 within $0.25 \mathrm{ml}$ PBS and inoculated peritoneally. Some mice were administered i.p with AG490 (500 ug/100 ul/mouse) or equivalent concentration of DMSO once a week. All mice were sacrificed after 30 days and peritoneal metastasis nodules were counted. All animal studies were conducted with the approval of the Committee on Animal Care in Shanghai Jiao Tong University School of Medicine.

\section{Statistical analysis}

Results were summarized as means $\pm \mathrm{SD}$. Student $t$ test and one-way analysis of variance (ANOVA) was used to analyze the data and the significance level was set at $P<0.05$.

\section{CONFLICTS OF INTEREST}

The authors made no disclosures.

\section{GRANT SUPPORT}

This study was supported by grants from National Natural Science Foundation of China (No.81272749, No.81572798 and No.91529302), and Shanghai Municipal Education Commission-Gaofeng Clinical Medicine Grant Support (20152505).

\section{REFERENCES}

1. Torre LA, Bray F, Siegel RL, Ferlay J, Lortet-Tieulent J, Jemal A. Global cancer statistics, 2012. CA Cancer J Clin. 2015; 65:87-108.

2. Moon YW, Jeung HC, Rha SY, Yoo NC, Roh JK, Noh SH, Kim BS, Chung HC. Changing patterns of prognosticators during 15-year follow-up of advanced gastric cancer after radical gastrectomy and adjuvant chemotherapy: a 15year follow-up study at a single korean institute. Annals of surgical oncology. 2007; 14:2730-2737.
3. Thiery JP. Epithelial-mesenchymal transitions in tumour progression. Nature reviews Cancer. 2002; 2:442-454.

4. Yang J, Weinberg RA. Epithelial-mesenchymal transition: at the crossroads of development and tumor metastasis. Developmental cell. 2008; 14:818-829.

5. Zeisberg M, Neilson EG. Biomarkers for epithelialmesenchymal transitions. J Clin Invest. 2009; 119:1429-1437.

6. Zheng X, Carstens JL, Kim J, Scheible M, Kaye J, Sugimoto H, Wu CC, LeBleu VS, Kalluri R. Epithelial-tomesenchymal transition is dispensable for metastasis but induces chemoresistance in pancreatic cancer. Nature. 2015; 527:525-530.

7. Bae YK, Choi JE, Kang SH, Lee SJ. EpithelialMesenchymal Transition Phenotype Is Associated with Clinicopathological Factors That Indicate Aggressive Biological Behavior and Poor Clinical Outcomes in Invasive Breast Cancer. J Breast Cancer. 2015; 18:256-263.

8. Elliott BE, Hung WL, Boag AH, Tuck AB. The role of hepatocyte growth factor (scatter factor) in epithelialmesenchymal transition and breast cancer. Can J Physiol Pharmacol. 2002; 80:91-102.

9. Massague J. TGFbeta in Cancer. Cell. 2008; 134:215-230.

10. Hu TH, Yao Y, Yu S, Han LL, Wang WJ, Guo H, Tian T, Ruan ZP, Kang XM, Wang J, Wang SH, Nan KJ. SDF-1/ CXCR4 promotes epithelial-mesenchymal transition and progression of colorectal cancer by activation of the Wnt/betacatenin signaling pathway. Cancer letters. 2014; 354:417-426.

11. Du R, Xia L, Ning X, Liu L, Sun W, Huang C, Wang H, Sun S. Hypoxia-induced Bmil promotes renal tubular epithelial cell-mesenchymal transition and renal fibrosis via PI3K/Akt signal. Mol Biol Cell. 2014; 25:2650-2659.

12. Kalluri R, Zeisberg M. Fibroblasts in cancer. Nature reviews Cancer. 2006; 6:392-401.

13. Cirri P, Chiarugi P. Cancer associated fibroblasts: the dark side of the coin. Am J Cancer Res. 2011; 1:482-497.

14. Wu X, Chen X, Zhou Q, Li P, Yu B, Li J, Qu Y, Yan J, Yu Y, Yan M, Zhu Z, Liu B, Su L. Hepatocyte growth factor activates tumor stromal fibroblasts to promote tumorigenesis in gastric cancer. Cancer letters. 2013; 335:128-135.

15. Nagasaki T, Hara M, Nakanishi H, Takahashi H, Sato M, Takeyama H. Interleukin-6 released by colon cancerassociated fibroblasts is critical for tumour angiogenesis: anti-interleukin-6 receptor antibody suppressed angiogenesis and inhibited tumour-stroma interaction. Br J Cancer. 2014; 110:469-478.

16. Shiga K, Hara M, Nagasaki T, Sato T, Takahashi H, Takeyama H. Cancer-Associated Fibroblasts: Their Characteristics and Their Roles in Tumor Growth. Cancers. 2015; 7:2443-2458.

17. Grivennikov SI, Greten FR, Karin M. Immunity, inflammation, and cancer. Cell. 2010; 140:883-899.

18. Culig Z, Puhr M. Interleukin-6: a multifunctional targetable cytokine in human prostate cancer. Molecular and cellular endocrinology. 2012; 360:52-58. 
19. Waldner MJ, Foersch S, Neurath MF. Interleukin-6 - a key regulator of colorectal cancer development. Int J Biol Sci. 2012; 8:1248-1253.

20. Bromberg J, Wang TC. Inflammation and cancer: IL-6 and STAT3 complete the link. Cancer cell. 2009; 15:79-80.

21. Banerjee K, Resat H. Constitutive activation of STAT3 in breast cancer cells: A review. Int J Cancer. 2016; 138:2570-2578.

22. Liu RY, Zeng Y, Lei Z, Wang L, Yang H, Liu Z, Zhao J, Zhang HT. JAK/STAT3 signaling is required for TGF-betainduced epithelial-mesenchymal transition in lung cancer cells. Int J Oncol. 2014; 44:1643-1651.

23. Wang SW, Sun YM. The IL-6/JAK/STAT3 pathway: potential therapeutic strategies in treating colorectal cancer (Review). Int J Oncol. 2014; 44:1032-1040.

24. Jackson CB, Judd LM, Menheniott TR, Kronborg I, Dow C, Yeomans ND, Boussioutas A, Robb L, Giraud AS. Augmented gp130-mediated cytokine signalling accompanies human gastric cancer progression. J Pathol. 2007; 213:140-151.

25. Zhang XM, Zhou C, Gu H, Yan L, Zhang GY. Correlation of RKIP, STAT3 and cyclin D1 expression in pathogenesis of gastric cancer. Int J Clin Exp Pathol. 2014; 7:5902-5908.

26. De Vlieghere E, Verset L, Demetter P, Bracke M, De Wever O. Cancer-associated fibroblasts as target and tool in cancer therapeutics and diagnostics. Virchows Arch. 2015; 467:367-382.

27. Benwell RK, Lee DR. Essential and synergistic roles of IL1 and IL6 in human Th17 differentiation directed by TLR ligand-activated dendritic cells. Clinical immunology. 2010; 134:178-187.

28. Kinoshita H, Hirata $Y$, Nakagawa H, Sakamoto K, Hayakawa Y, Takahashi R, Nakata W, Sakitani K, Serizawa T, Hikiba Y, Akanuma M, Shibata W, Maeda S, et al. Interleukin-6 mediates epithelial-stromal interactions and promotes gastric tumorigenesis. PloS one. 2013; 8:e60914.

29. Bhowmick NA, Neilson EG, Moses HL. Stromal fibroblasts in cancer initiation and progression. Nature. 2004; 432:332-337.

30. Erez N, Truitt M, Olson P, Arron ST, Hanahan D. CancerAssociated Fibroblasts Are Activated in Incipient Neoplasia to Orchestrate Tumor-Promoting Inflammation in an NFkappaB-Dependent Manner. Cancer cell. 2010; 17:135-147.
31. Li P, Shan JX, Chen XH, Zhang D, Su LP, Huang XY, Yu BQ, Zhi QM, Li CL, Wang YQ, Tomei S, Cai Q, $\mathrm{Ji}$, et al. Epigenetic silencing of microRNA-149 in cancer-associated fibroblasts mediates prostaglandin E2/ interleukin-6 signaling in the tumor microenvironment. Cell research. 2015; 25:588-603.

32. Yadav A, Kumar B, Datta J, Teknos TN, Kumar P. IL-6 promotes head and neck tumor metastasis by inducing epithelial-mesenchymal transition via the JAK-STAT3-SNAIL signaling pathway. Mol Cancer Res. 2011; 9:1658-1667.

33. Kim MS, Lee WS, Jeong J, Kim SJ, Jin W. Induction of metastatic potential by TrkB via activation of IL6/ JAK2/STAT3 and PI3K/AKT signaling in breast cancer. Oncotarget. 2015; 6:40158-40171. doi: 10.18632/ oncotarget.5522.

34. Galdiero M, Vitiello M, D'Isanto M, Raieta K, Galdiero E. STAT1 and STAT3 phosphorylation by porins are independent of JAKs but are dependent on MAPK pathway and plays a role in U937 cells production of interleukin-6. Cytokine. 2006; 36:218-228.

35. Yang F, Tuxhorn JA, Ressler SJ, McAlhany SJ, Dang TD, Rowley DR. Stromal expression of connective tissue growth factor promotes angiogenesis and prostate cancer tumorigenesis. Cancer research. 2005; 65:8887-8895.

36. Augsten M, Hagglof C, Olsson E, Stolz C, Tsagozis P, Levchenko T, Frederick MJ, Borg A, Micke P, Egevad L, Ostman A. CXCL14 is an autocrine growth factor for fibroblasts and acts as a multi-modal stimulator of prostate tumor growth. Proc Natl Acad Sci USA. 2009; 106:3414-3419.

37. Orimo A, Gupta PB, Sgroi DC, Arenzana-Seisdedos F, Delaunay T, Naeem R, Carey VJ, Richardson AL, Weinberg RA. Stromal fibroblasts present in invasive human breast carcinomas promote tumor growth and angiogenesis through elevated SDF-1/CXCL12 secretion. Cell. 2005; 121:335-348.

38. Semerano L, Thiolat A, Minichiello E, Clavel G, Bessis N, Boissier MC. Targeting IL-6 for the treatment of rheumatoid arthritis: Phase II investigational drugs. Expert Opin Investig Drugs. 2014; 23:979-999. 